\title{
Quantum oscillations of the critical current and high-field superconducting proximity in ballistic graphene
}

\author{
M. Ben Shalom ${ }^{1,2}$, M. J. Zhu ${ }^{1,2}$, V. I. Fal'ko ${ }^{1,2 \star}$, A. Mishchenko ${ }^{1,2}$, A. V. Kretinin', K. S. Novoselov ${ }^{1}$, \\ C. R. Woods ${ }^{2}$, K. Watanabe ${ }^{3}$, T. Taniguchi ${ }^{3}$, A. K. Geim ${ }^{1,2 \star}$ and J. R. Prance ${ }^{4}$
}

\begin{abstract}
Graphene-based Josephson junctions provide a novel platform for studying the proximity effect ${ }^{1-3}$ due to graphene's unique electronic spectrum and the possibility to tune junction properties by gate voltage $e^{4-16}$. Here we describe graphene junctions with a mean free path of several micrometres, low contact resistance and large supercurrents. Such devices exhibit pronounced Fabry-Pérot oscillations not only in the normal-state resistance but also in the critical current. The proximity effect is mostly suppressed in magnetic fields below $10 \mathrm{mT}$, showing the conventional Fraunhofer pattern. Unexpectedly, some proximity survives even in fields higher than $1 \mathrm{~T}$. Superconducting states randomly appear and disappear as a function of field and carrier concentration, and each of them exhibits a supercurrent carrying capacity close to the universal quantum limit ${ }^{17,18}$. We attribute the high-field Josephson effect to mesoscopic Andreev states that persist near graphene edges. Our work reveals new proximity regimes that can be controlled by quantum confinement and cyclotron motion.
\end{abstract}

The superconducting proximity effect relies on penetration of Cooper pairs from a superconductor (S) into a normal metal (N) and is most pronounced in systems with transparent SN interfaces and weak scattering, where superconducting correlations penetrate deep inside the normal metal. Despite being one atom thick and having a low density of states, which vanishes at the Dirac point, graphene $(G)$ can exhibit low contact resistance and ballistic transport on a micrometre scale ${ }^{19,20}$ exceeding the distance between the superconducting leads by an order of magnitude. These properties combined with the possibility to electrostatically control the carrier density $n$ offer tunable Josephson junctions in a regime that can be referred to as ballistic proximity superconductivity ${ }^{21}$. Despite intense interest in SGS devices ${ }^{3-16}$ that can show features qualitatively different from the conventional SNS behaviour ${ }^{2,3}$, ballistic graphene Josephson junctions ${ }^{15,16}$ remain little studied.

Our SGS devices are schematically shown in Fig. 1 and described in further detail in Supplementary Section 1. The essential technological difference from the previously studied SGS junctions ${ }^{4-14}$ is the use of graphene encapsulated between boron nitride crystals ${ }^{19,20}$ as well as a new nanostrip geometry of the contacts. This allows high carrier mobility, low charge inhomogeneity and low contact resistance. More than twenty SGS junctions with a width $W$ between 3 and $8 \mu \mathrm{m}$ and a length $L$ between 0.15 and $2.5 \mu \mathrm{m}$ were studied, all exhibiting a finite supercurrent at low temperatures $(T)$, reproducible behaviour and consistent changes with $L$ and $W$. First, we characterize the devices above the transition temperature $T_{\mathrm{C}} \approx 7 \mathrm{~K}$ of our superconducting contacts. Figure $1 \mathrm{~b}$ shows examples of the normal-state resistance $R_{\mathrm{n}}$ as a function of back gate voltage $V_{\mathrm{g}}$ that changes $n$ in graphene. The neutrality point (NP) was found to be shifted to negative $V_{\mathrm{g}}$ by a few volts, with the shift being consistently larger for shorter devices (Supplementary Information). This is due to electron doping induced by our $\mathrm{Nb}$ contacts. For ballistic graphene such doping is practically uniform away from the metal interface ${ }^{22}$. The observed smearing of $R_{\mathrm{n}}\left(V_{\mathrm{g}}\right)$ curves near the NP allows an estimate for charge inhomogeneity in the graphene bulk of $\approx 2 \times 10^{10} \mathrm{~cm}^{-2}$. For consistency, data for devices with different $L$ are presented as a function of $\Delta V_{\mathrm{g}}$, the gate voltage counted from the NP.

For positive $\Delta V_{\mathrm{g}}$ (electron doping) and $n>10^{11} \mathrm{~cm}^{-2}$, SGS junctions made from the same graphene crystal and having the same $W$ exhibit the same $R_{\mathrm{n}}\left(\Delta V_{\mathrm{g}}\right)$ dependence, independently of $L$ (Fig. 1b). This shows that the mean free path is larger than the contact separation and yields carrier mobility $>300,000 \mathrm{~cm}^{2} \mathrm{~V}^{-1} \mathrm{~s}^{-1}$, in agreement with the quality measured for similarly made Hall bar devices. The dashed curve in Fig. $1 \mathrm{~b}$ indicates the behaviour expected in the quantum ballistic limit $R_{\mathrm{Q}}=\left(h / e^{2}\right) / 4 N$ where $N=\operatorname{int}\left(2 W / \lambda_{\mathrm{F}}\right)$ is the number of propagating electron modes, $\lambda_{\mathrm{F}}$ the Fermi wavelength that depends on $n\left(\Delta V_{\mathrm{g}}\right)$ and the factor 4 corresponds to graphene's degeneracy. The difference between $R_{\mathrm{Q}}$ and the experimental curves yields a record low contact resistivity, $\approx 35 \Omega \mu \mathrm{m}$. This value corresponds to an angle-averaged transmission probability $\operatorname{Tr} \approx 0.8$ (Supplementary Section 2).

For hole doping, $R_{\mathrm{n}}$ becomes significantly higher indicating smaller $\operatorname{Tr}$. This is because $\mathrm{p}-\mathrm{n}$ junctions appear at the $\mathrm{Nb}$ contacts and lead to partial reflection of electron waves, which effectively creates a Fabry-Pérot (FP) cavity $^{5,23}$. The standing waves lead to pronounced oscillations in $R_{\mathrm{n}}$ as a function of both $V_{\mathrm{g}}$ and applied bias $V_{\mathrm{b}}$ (Fig. 1b). The oscillatory behaviour indicates that charge carriers can cross the graphene strip several times, preserving their monochromaticity and coherence. Some FP oscillations could also be discerned for positive $\Delta V_{\mathrm{g}}$ but they were much weaker because of higher $\mathrm{Tr}$. The observed FP behaviour in the normal state agrees with earlier reports ${ }^{5,23}$. Its details can be modelled accurately if we take into account that the position of $\mathrm{p}-\mathrm{n}$ junctions varies with $V_{\mathrm{g}}$ so that the effective length of the FP interferometer becomes notably shorter than $L$ at low hole doping (Supplementary Section 3 ).

After characterizing our SGS devices at $T>T_{\mathrm{C}}$, we turn to their superconducting behaviour. All of the junctions (including

\footnotetext{
${ }^{1}$ National Graphene Institute, The University of Manchester, Booth St. E, Manchester M13 9PL, UK. ${ }^{2}$ School of Physics and Astronomy, The University of Manchester, Manchester M13 9PL, UK. ${ }^{3}$ National Institute for Materials Science, 1-1 Namiki, Tsukuba 305-0044, Japan. ${ }^{4}$ Department of Physics, University of Lancaster, Lancaster LA1 4YW, UK. *e-mail: v.falko@lancaster.ac.uk; geim@man.ac.uk
} 

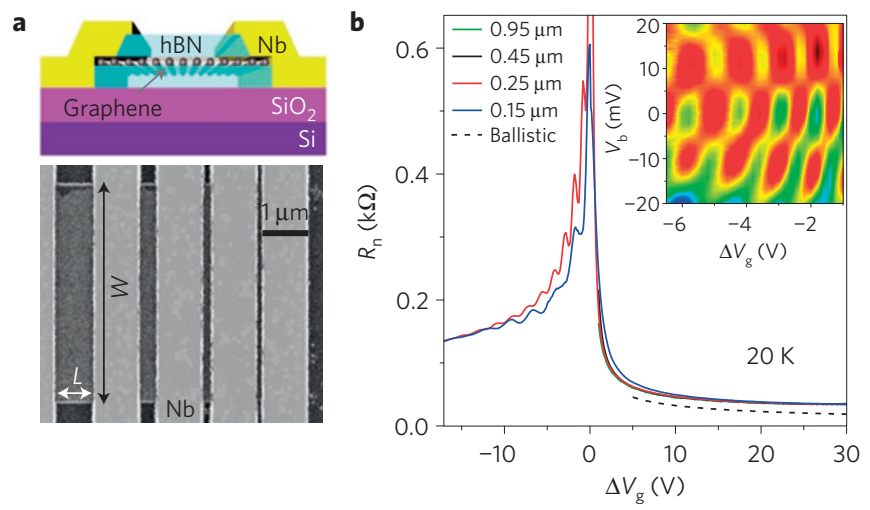

Figure 1 | Ballistic SGS junctions. a, Top: Junctions schematic. Bottom: Electron micrograph of a set of four junctions with different $L$. The few $\mathrm{nm}$-wide graphene ledge (top drawing) is referred to as a nanostrip contact. b. Typical behaviour for SGS junctions with different $L$ but for the same set of junctions with $W=5 \mu \mathrm{m}$. To avoid an obscuring overlap between four oscillating curves, we plot $R_{\mathrm{n}}$ at negative $\Delta V_{\mathrm{g}}$ only for the two shortest junctions. For positive $\Delta V_{g}>5 V$, the four curves overlap within the line width. The dashed curve shows calculated $R_{Q}(n)$. Inset: changes in the differential conductance $\mathrm{d} / \mathrm{dV} ; L=0.25 \mu \mathrm{m}$. Colour scale, -1 to $1 \mathrm{mS}$ (red to blue).

$L=2.5 \mu \mathrm{m}$ ) exhibited the fully developed proximity effect. Figure 2a,b show that the critical current $I_{c}$ remained finite at the NP and rapidly increased with $\left|\Delta V_{\mathrm{g}}\right|$, reaching densities $>5 \mu \mathrm{A} \mu \mathrm{m}^{-1}$ for high electron doping and short $L$, notably larger than values of $I_{c}$ previously reported ${ }^{4-16}$. Such high $I_{c}$ values are due to ballistic transport and low contact resistance. Indeed, $I_{\mathrm{c}}$ can theoretically reach a value $e^{2,24}$

$$
I_{\mathrm{c}}=\alpha \Delta / e R_{\mathrm{n}}
$$

where $\Delta$ is the superconducting gap and $\alpha \approx 2.1$. Because in our devices $R_{\mathrm{n}} \approx R_{\mathrm{Q}}$, the equation implies that we approach the quantum limit $I_{c} \approx(e \Delta / h) 4 N$ where the supercurrent is determined by the number of propagating electronic modes that transfer Cooper pairs between superconducting contacts, and each of the modes has the supercurrent carrying capacity,17 $I_{\mathrm{Q}} \approx e \Delta / h$.

Equation (1) suggests that $I_{\mathrm{c}} R_{\mathrm{n}}$ should be a constant. This holds well in our SGS devices away from the NP (Fig. 2c) and indicates that at low $T$, external noise, fluctuations and other mechanisms ${ }^{4-16}$ that are dependent on $n$ or $R_{\mathrm{n}}$ do not limit $I_{\mathrm{c}}$. However, Fig. $2 \mathrm{c}$ yields $\alpha$ that is notably smaller than the expected constant in equation (1). For hole doping, this can be attributed to the presence of $\mathrm{p}-\mathrm{n}$ junctions at the superconducting interfaces but even for electron doping and high $\operatorname{Tr}$ we find $\alpha \approx 0.4$ (Fig. 2c). Furthermore, we measured the excess current $I_{\mathrm{e}}>I_{\mathrm{c}}$ as shown in Fig. 2a and found that, in the case of $I_{\mathrm{e}}, \alpha$ also does not reach a value close to 2.1 (Fig. 2c). This corresponds to the fact that all our devices were in the limit of long $L>\Delta / h v_{\mathrm{F}}$ ( $v_{\mathrm{F}}$ is the Fermi velocity), as also follows from the $1 / L$ dependence found for $I_{\mathrm{c}} R_{\mathrm{n}}$ (Fig. $2 \mathrm{~d}$ ). In this long-junction regime, the critical current is given by $I_{\mathrm{c}} \approx E_{\mathrm{Th}} / e R_{\mathrm{n}}$ being determined by the Thouless energy $E_{\mathrm{Th}}$ rather than the superconducting gap ${ }^{2,17,24}$. For a ballistic system, $E_{\mathrm{Th}}$ depends on the time that the charge carriers spent inside the FP cavity and can be estimated $^{25}$ as $\sim h v_{\mathrm{F}} / L$. This yields $I_{\mathrm{c}} \propto 1 / L$ and $I_{\mathrm{c}} R_{\mathrm{n}} \propto 1 / L$ because $R_{\mathrm{n}}$ is independent of the ballistic device's length. Our detailed studies of $I_{\mathrm{c}}$ as a function of $T$ and $L$ show that all data for $I_{\mathrm{c}} R_{\mathrm{n}} / E_{\mathrm{Th}}$ collapse on a universal curve $f\left(T / E_{\mathrm{Th}}\right)$ with $E_{\mathrm{Th}} \sim h v_{\mathrm{F}} / L$, which again agrees well with expectations for the long-junction limit (Supplementary Section 4 ). We estimate that to reach the transition regime $E_{\mathrm{Th}} / \Delta \sim 1$ for our SGS junctions would require $L<100 \mathrm{~nm}$. Let us also mention that no definitive signs of specular Andreev reflection ${ }^{3,17}$ were found in our devices (Supplementary Section 4).

As a consequence of FP resonances in the normal state (Fig. 1), the supercurrent also exhibits quantum oscillations that are clearly seen in Fig. $2 \mathrm{~b}$ for hole doping. Equation (1) implies that such oscillations in $I_{\mathrm{c}}$ should occur simply because $R_{\mathrm{n}}$ oscillates. Indeed, $R_{\mathrm{n}}$ and $I_{\mathrm{c}}$ are found to oscillate in antiphase, compensating each other in the final products $I_{c, e} R_{\mathrm{n}}$. However, we find that oscillations in the critical current are approximately 3 times stronger than those in $R_{\mathrm{n}}$. This observation is consistent with the fact that $I_{\mathrm{c}}$ is not only inversely proportional to $R_{\mathrm{n}}$ but also depends on the Thouless energy as discussed above, whereas the latter is expected to oscillate because of the oscillating transparency of FP resonators (Supplementary Section 4). We note in passing that $R_{\mathrm{n}}$ and its FP oscillations exhibit little temperature dependence below $20 \mathrm{~K}$, which justifies the use of $R_{\mathrm{n}}$ measured above $T_{\mathrm{C}}$ in the above analysis of the superconducting behaviour.

In a magnetic field $B$, our ballistic junctions exhibit further striking departures from the conventional behaviour (Fig. 3). In small $B$ such that a few flux quanta $\phi_{0}=h / 2 e$ enter an SGS junction, we observe the standard Fraunhofer dependence ${ }^{2}$

$$
I_{\mathrm{c}}(B)=I_{\mathrm{c}}(B=0)\left|\sin \left(\pi \Phi / \phi_{0}\right) /\left(\pi \Phi / \phi_{0}\right)\right|
$$

where $\Phi=L \times W \times B$ is the flux through the junction area. Marked deviations from equation (2) occur in $B>5 \mathrm{mT}$ (Fig. 3a). Figure $3 \mathrm{~b}-\mathrm{e}$ shows that, in this regime, the supercurrent no longer follows the oscillatory Fraunhofer pattern but pockets of proximity superconductivity can randomly appear as a function of $n$ and $B$. At low doping, the pockets can be separated by extended regions of the normal state where no supercurrent could be detected with accuracy of a few $\mathrm{nA} \ll I_{\mathrm{Q}}$ (Fig. 3c,e). Within each pocket, $I-V$ characteristics exhibit a gapped behaviour (inset of Fig. 3d) with $I_{\mathrm{c}} \sim I_{\mathrm{Q}} \approx 40 \mathrm{nA}$, although the exact value depends on doping and $I_{c}$ decreases to $\approx 10 \mathrm{nA}$ close to the NP, possibly due to the rising contributions of electrical noise and thermal fluctuations that suppress apparent $I_{c}$ (Fig. 3c). These proximity states persist up to $B \approx 1 \mathrm{~T}\left(\Phi / \phi_{0} \sim 10^{3}\right)$ and are highly reproducible, although occasional flux jumps in the $\mathrm{Nb}$ contacts can reset the proximity pattern (Supplementary Section 5). The correlation analysis presented in Supplementary Section 6 indicates that to suppress such superconducting states, changes in $\Phi$ of $\approx \phi_{0}$ and changes in the Fermi energy of $\approx 1 \mathrm{meV}$ are required.

The semiclassical description ${ }^{2,24-27}$ of the superconducting proximity relates the Cooper pair transfer between the leads to electrons and Andreev-reflected holes, which travel along same trajectories but in opposite directions (Fig. 3f). In low $B$, interference between many Andreev states traversing the graphene strip results in the Fraunhofer-type oscillatory suppression of $I_{c}$ described by equation (2) (see Fig. 3a). Although not reported before, the Fraunhofer pattern in ballistic devices can be expected to break down in relatively small $B$ because the cyclotron motion deflects electrons and holes in opposite directions so that they can no longer retrace each other (Fig. 3g). We have estimated the field required to suppress Andreev states in the bulk as $B^{*} \sim \Delta / e L v_{\mathrm{F}}$ (Supplementary Section 7). For the devices in Fig. 3, this yields $B^{*} \approx 5 \mathrm{mT}$, in agreement with the field where strong deviations from the Fraunhofer curve are observed.

As for the random pockets of superconductivity at $B \gg B^{*}$ that exhibit $I_{c}$ much higher than that expected from equation (2), we invoke the previously noticed analogy ${ }^{18}$ between mesoscopic fluctuations in the normal-state conductance ${ }^{28},\left\langle\delta G^{2}\right\rangle$ and in the supercurrent ${ }^{17,18},\left\langle\delta I_{c}^{2}\right\rangle$. Both types of fluctuations are due to interference of electron waves propagating along different paths but start and finish together. In contrast to the case of $B=0$, for which semiclassical phases of counter-propagating electrons and 

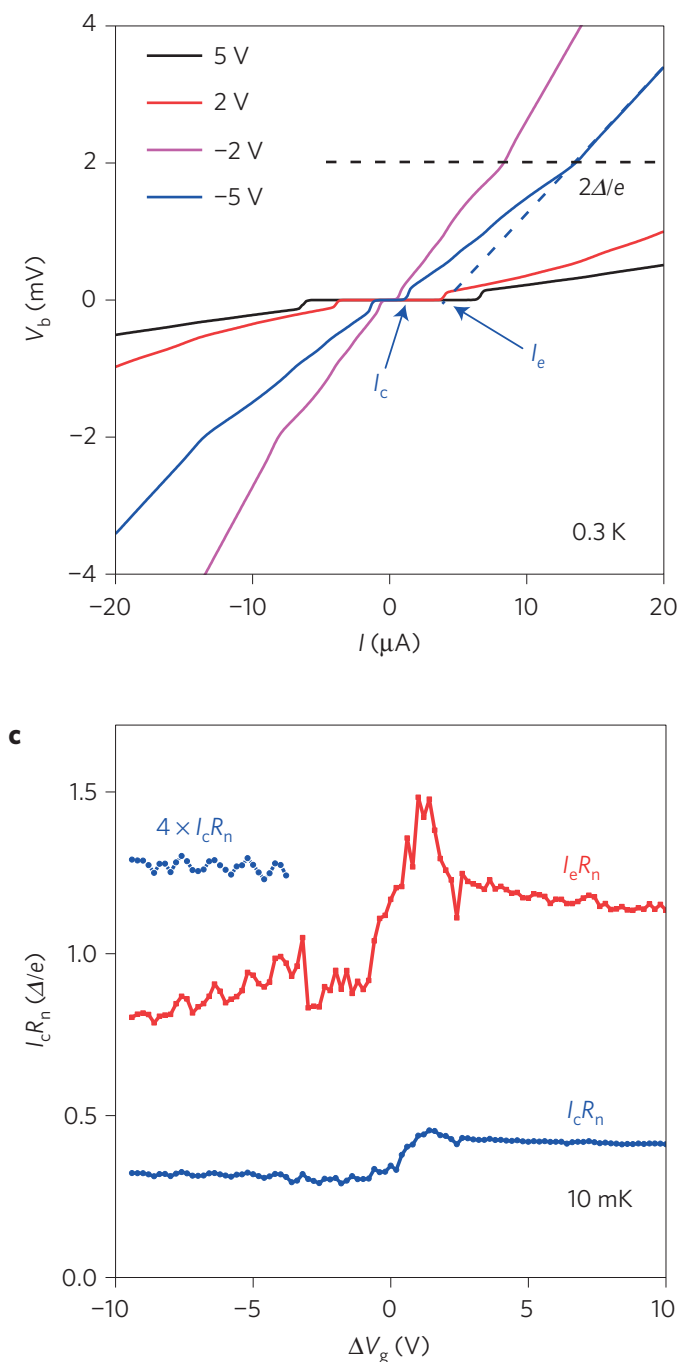

b

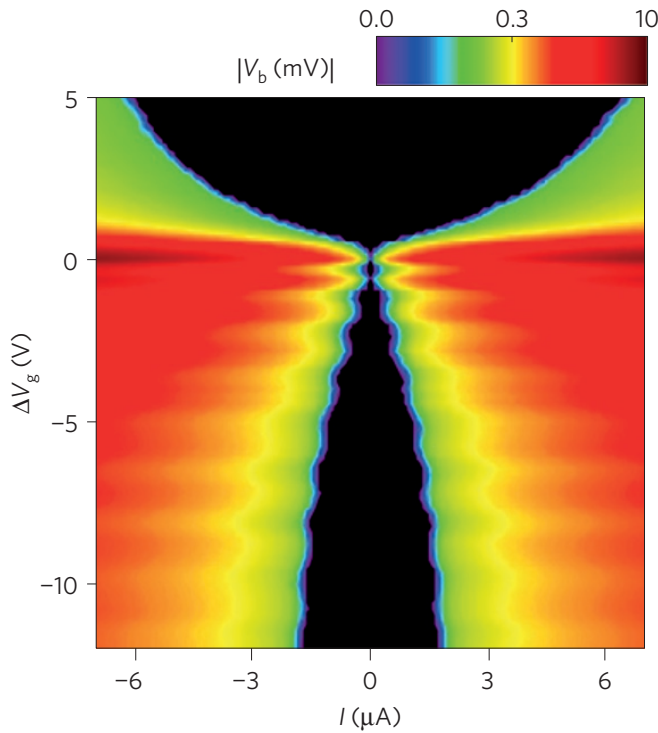

d

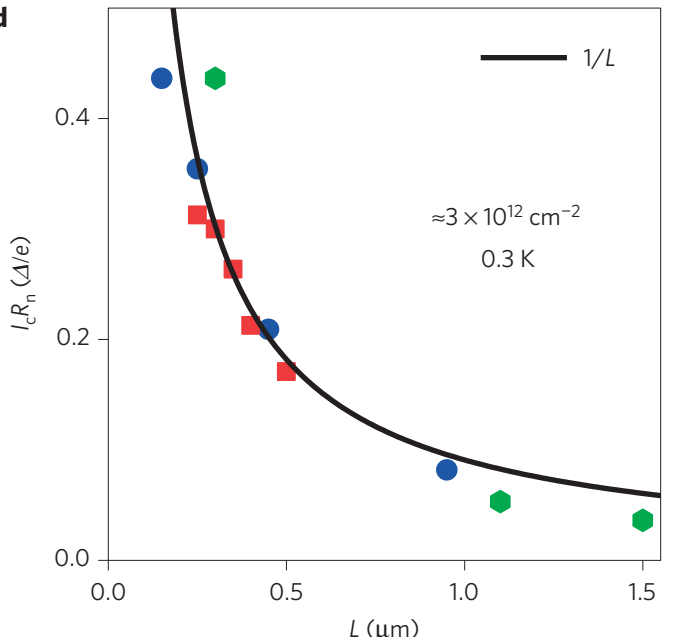

Figure 2 | Quantum oscillations in the supercurrent. a, Examples of $I-V$ characteristics for ballistic SGS junctions in the superconducting state. The data are for the device in Fig. 1 with $L=0.25 \mu \mathrm{m}$. The arrows explain notions $I_{\mathrm{c}}$ and $I_{\mathrm{e}}$. $\mathbf{b}$, Absolute voltage drop $\left|V_{\mathrm{b}}\right|$ across the SGS junction in (a) for a wide range of doping. The black region corresponds to the zero-resistance state, and its edge exhibits clear FP oscillations. c, $I_{\mathrm{c}} R_{\mathrm{n}}$ and $I_{\mathrm{e}} R_{\mathrm{n}}$ for a device with $L=0.3 \mu \mathrm{m}$, $W=6.5 \mu \mathrm{m}$ and $\Delta \approx 1.0 \mathrm{meV}$ estimated from its $T_{\mathrm{C}}$. Each data point is extracted from a trace such as in $(\mathbf{a})$. Inset: Oscillatory part of $I_{\mathrm{C}} R_{\mathrm{n}}$ is magnified. Similar behaviour was observed for other devices. $\mathbf{d}$, Effect of the junction length on supercurrent for 12 devices with different $W$. Red symbols, $W=3 \mu \mathrm{m}$; blue, $5 \mu \mathrm{m}$; green, $6.5 \mu \mathrm{m}$. For each data set, $I_{\mathrm{c}}$ follows the same dependence as $I_{\mathrm{c}} R_{\mathrm{n}}$ because $R_{\mathrm{n}}$ were practically independent of $L$ for the same $W$. For the two longest devices in (d), the critical current falls below the plotted 1/L dependence, probably because of thermal fluctuations (Supplementary Section 4 ).

holes near the Fermi level cancel each other because of time-reversal symmetry (Fig. 3f), electrons and holes propagating along nonretracing trajectories in a finite $B$ acquire large and random phase differences (Fig. 3g,h). Averaging over all imaginable geometrical paths would lead to the complete suppression of the supercurrent ${ }^{18}$. However, for each given realization of either diffusive or chaotic ballistic SNS junctions, the characteristic values of fluctuations are $\operatorname{set}^{17,18,28-30}$ at $\left\langle\delta G^{2}\right\rangle^{1 / 2} \sim e^{2} / h$ and $\left\langle\delta I_{c}^{2}\right\rangle^{1 / 2} \sim e \Delta / h$. In the case of $B \gg B^{*}$, non-retracing paths that can transfer Cooper pairs between superconducting contacts can occur only near graphene edges (see Fig. 3h and Supplementary Section 7). In a way, a combination of cyclotron motion and edge scattering provides a chaotic ballistic billiard near each graphene edge, and this leads to random pockets of superconductivity with $I_{\mathrm{c}}=\left\langle\delta I_{\mathrm{c}}^{2}\right\rangle^{1 / 2} \sim I_{\mathrm{Q}}$. Moreover, the analogy with chaotic billiards allows us to estimate the change in the Fermi energy, which is needed to change the realization of the mesoscopic system and, therefore, suppress an existing pocket of superconductivity. The required change is again given by the Thouless energy $E_{\mathrm{Th}} \sim h v_{\mathrm{F}} / \Lambda$ where $\Lambda$ is the typical length of Andreev paths in a strong magnetic field (Fig. $3 \mathrm{~h}$ ). At high $B$, we estimate $\Lambda$ as $\approx\left(r_{\mathrm{c}} L\right)^{1 / 2}$ where $r_{\mathrm{c}}$ is the cyclotron radius. This yields $E_{\mathrm{Th}} \leq 1 \mathrm{meV}$, in agreement with the observed changes in doping that are required to suppress the pockets of superconducting proximity (Supplementary Section 6). An interference pattern in mesoscopic systems is also known ${ }^{18,28-30}$ to change upon changing the flux $\Phi$ through the system by $\approx \phi_{0}$. This scale agrees well with that observed experimentally (Supplementary Figure 8)

Finally, the discussed mesoscopic proximity effect can be expected to disappear if $r_{c}$ becomes shorter than $L / 2$ (Fig. 3i). This condition is marked in Fig. $3 \mathrm{~d}$ and seen more clearly in the data of Supplementary Section 8. It is also worth noting that the near-edge superconductivity was not observed for hole doping, which we attribute to the fact that Klein tunnelling in graphene collimates trajectories perpendicular to the $\mathrm{p}-\mathrm{n}$ interface $\mathrm{e}^{23}$, making 
a

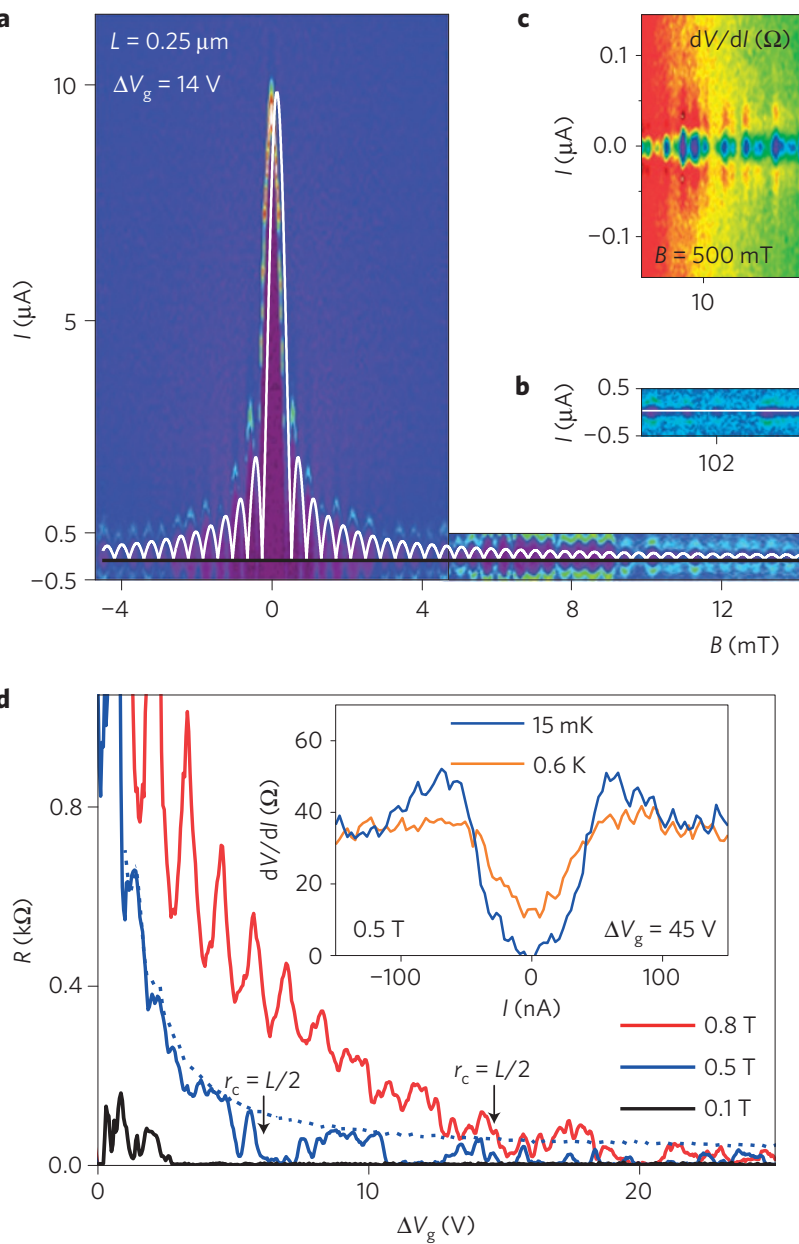

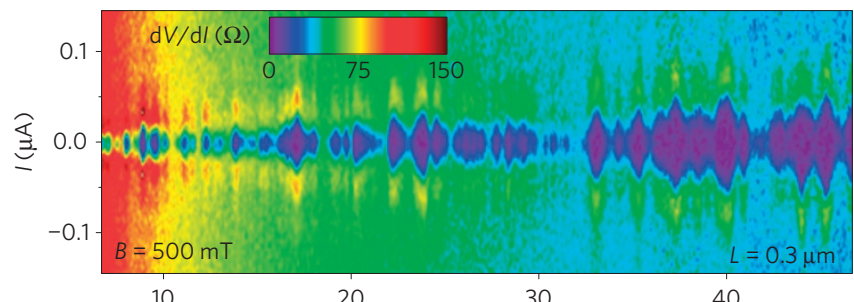

20 $\Delta V_{g}(V)$
$30 \quad 40$

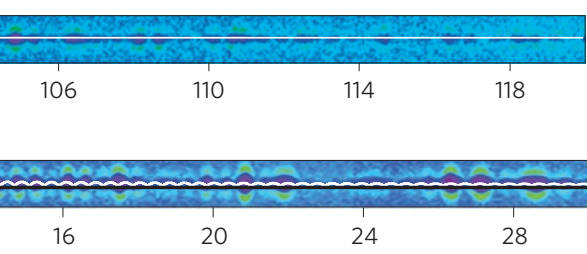

e

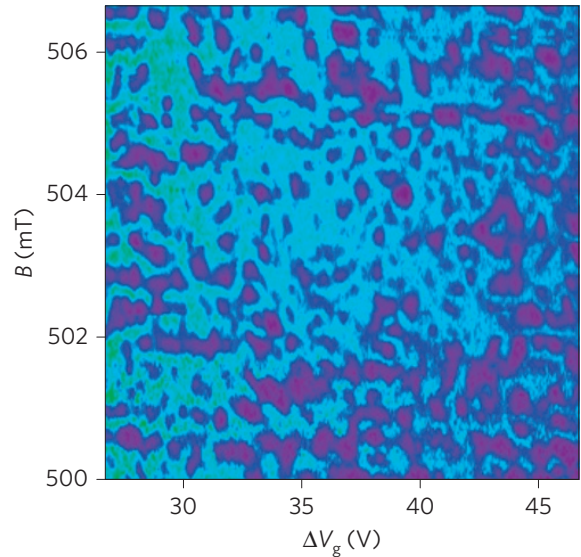

$\mathbf{f}$

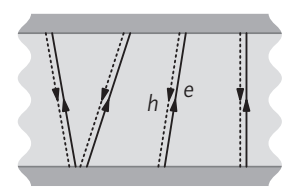

g

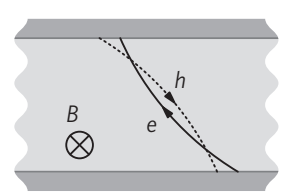

h

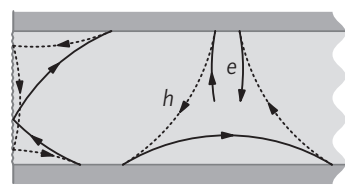

i

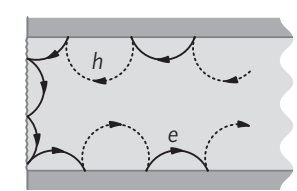

Figure 3 | Fluctuating proximity superconductivity. a, Example of $\mathrm{dV} / \mathrm{d} /$ as a function of applied current $I$ and $B$. The purple regions correspond to the zero-resistance state and their edges mark $I_{c}$ (see Supplementary Section 10). The map is symmetric in both I and $B$. The white curve is given by equation (2). The low- $B$ periodicity is $\approx 0.4 \mathrm{mT}$, smaller than expected from the device's area, which is attributed to the Meissner screening that focuses the field into the junction ${ }^{30} . \mathbf{b}$, Continuation of the map from (a) above 0.1 T. Intervals with finite $I_{\mathrm{c}}$ continue to randomly appear, despite the Fraunhofer curve being indistinguishable from zero. c, Another high- $B$ example but as a function of $\Delta V_{\mathrm{g}}$ in $0.5 \mathrm{~T}$. d, Examples of low-current resistance $(I=2 \mathrm{nA})$ in different $B$. The dashed curve for $0.5 \mathrm{~T}$ shows that current $I=150 \mathrm{nA}>I_{\mathrm{Q}}$ completely suppresses superconductivity. The arrows mark the expected onset of edge state transport. Inset: Typical $I-V$ characteristics for high- $B$ superconducting states. e, Local map of fluctuating superconductivity. $T \approx 10 \mathrm{mK}$; all colour scales are as in $\mathbf{c}$. $\mathbf{f}-\mathbf{i}$, Electron-hole paths responsible for Andreev states in ballistic junctions in zero (f), intermediate ( $(\mathbf{g}, \mathbf{h})$ and high $B(\mathbf{i})$. In (h), the cyclotron bending suppresses the transfer of Cooper pairs in the middle of the graphene strip but Andreev states can persist near the edges.

it essentially impossible to form the closed-loop Andreev states shown in Fig. 3h (Supplementary Section 7). In principle, the effect of near-edge Andreev states could be further enhanced by the presence of extended electronic states at graphene edges ${ }^{16}$ but, based on our experimental data, no evidence for this or other spatial inhomogeneity was found in the studied samples (Supplementary Section 9).

\section{Methods}

Methods and any associated references are available in the online version of the paper.

Received 15 April 2015; accepted 4 November 2015; published online 14 December 2015

\section{References}

1. Meissner, H. Range of order of superconducting electrons. Phys. Rev. Lett. 2, 458-459 (1959).

2. Tinkham, M. Introduction to Superconductivity (Courier Dover, 2012).

3. Beenakker, C. W. J. Colloquium: Andreev reflection and Klein tunneling in graphene. Rev. Mod. Phys. 80, 1337-1354 (2008).

4. Heersche, H. B., Jarillo-Herrero, P., Oostinga, J. B., Vandersypen, L. M. K. \& Morpurgo, A. F. Bipolar supercurrent in graphene. Nature 446, 56-59 (2007).

5. Miao, F. et al. Phase-coherent transport in graphene quantum billiards. Science 317, 1530-1533 (2007).

6. Du, X., Skachko, I. \& Andrei, E. Josephson current and multiple Andreev reflections in graphene SNS junctions. Phys. Rev. B 77, 184507 (2008).

7. Ojeda-Aristizabal, C., Ferrier, M., Guéron, S. \& Bouchiat, H. Tuning the proximity effect in a superconductor-graphene-superconductor junction. Phys. Rev. B 79, 165436 (2009).

8. Girit, C. et al. Tunable graphene dc superconducting quantum interference device. Nano Lett. 9, 198-199 (2009). 
9. Borzenets, I. V., Coskun, U. C., Jones, S. J. \& Finkelstein, G. Phase diffusion in graphene-based Josephson junctions. Phys. Rev. Lett. 107, 137005 (2011).

10. Komatsu, K., Li, C., Autier-Laurent, S., Bouchiat, H. \& Gueron, S Superconducting proximity effect through graphene from zero field to the quantum Hall regime. Phys. Rev. B 86, 115412 (2012).

11. Coskun, U. C. et al. Distribution of supercurrent switching in graphene under proximity effect. Phys. Rev. Lett. 108, 097003 (2012).

12. Mizuno, N., Nielsen, B. \& Du, X. Ballistic-like supercurrent in suspended graphene Josephson weak links. Nature Commun. 4, 3716 (2013).

13. Choi, J. H. et al. Complete gate control of supercurrent in graphene pn junctions. Nature Commun. 4, 3525 (2013).

14. Deon, F., Šopić, S. \& Morpurgo, A. F. Tuning the influence of microscopic decoherence on the superconducting proximity effect in a graphene Andreev interferometer. Phys. Rev. Lett. 112, 126803 (2014).

15. Calado, V. E. et al. Ballistic Josephson junctions in edge-contacted graphene. Nature Nanotech. 10, 761-764 (2015).

16. Allen, M. T. et al. Spatially resolved edge currents and guided-wave electronic states in graphene. Nature Phys. http://dx.doi.org/10.1038/nphys3534 (2015).

17. Beenakker, C. W. J. Universal limit of critical-current fluctuations in mesoscopic Josephson junctions. Phys. Rev. Lett. 67, 3836-3839 (1991).

18. Altshuler, B. L. \& Spivak, B. Z. Mesoscopic fluctuations in a superconductor-normal metal-superconductor junction. Sov. Phys. JETP 65, 343-347 (1987).

19. Mayorov, A. S. et al. Micrometer-scale ballistic transport in encapsulated graphene at room temperature. Nano Lett. 11, 2396-2399 (2011).

20. Wang, L. et al. One-dimensional electrical contact to a two-dimensional material. Science 342, 614-617 (2013).

21. Krylov, I. P. \& Sharvin, Y. V. Radio-frequency size effect in a layer of normal metal bounded by its superconducting phase. Sov. Phys. JETP 37, 481-486 (1973).

22. Blake, P. et al. Influence of metal contacts and charge inhomogeneity on transport properties of graphene near the neutrality point. Solid State Commun. 149, 1068-1071 (2009)

23. Rickhaus, P. et al. Ballistic interferences in suspended graphene. Nature Commun. 4, 2342 (2013).

24. Dubos, P. et al. Josephson critical current in a long mesoscopic SNS junction. Phys. Rev. B 63, 064502 (2001).
25. Ishii, C. Josephson currents through junctions with normal metal barriers. Prog. Theor. Phys. 44, 1525-1547 (1970).

26. Andreev, A. F. The thermal conductivity of the intermediate state in superconductors. Sov. Phys. JETP 19, 1228-1231 (1964).

27. Klapwijk, T. M. Proximity effect from an Andreev perspective. J. Supercond. 17, 593-611 (2004)

28. Jalabert, R. A., Baranger, H. U. \& Stone, A. D. Conductance fluctuations in the ballistic regime: A probe of quantum chaos? Phys. Rev. Lett. 65, 2442-2445 (1990)

29. Takayanagi, H., Hansen, J. B. \& Nitta, J. Mesoscopic fluctuations of the critica current in a superconductor-normal-conductor-superconductor. Phys. Rev. Lett. 74, 166-169 (1995).

30. Heida, J. P., van Wees, B. J., Klapwijk, T. M. \& Borghs, G. Nonlocal supercurrent in mesoscopic Josephson junctions. Phys. Rev. B 57, 5618-5621 (1998).

\section{Acknowledgements}

This work was supported by the European Research Council, the EU Graphene Flagship Program, the Royal Society, the Air Force Office of Scientific Research, the Office of Naval Research and ERC Synergy Grant Hetero2D. We thank C. Barton, G. H. Auton, R. V. Gorbachev and F. Guinea for helpful discussions. M.J.Z. acknowledges the National University of Defense Technology (China) overseas PhD scholarship. J.R.P. acknowledges support of the Marie Curie People Program.

\section{Author contributions}

A.K.G., A.V.K. and M.B.S. designed the experiment. M.B.S. and A.V.K. fabricated the devices. M.J.Z. and J.R.P. carried out the measurements. M.B.S., M.J.Z., V.I.F., A.K.G. and J.R.P. analysed and interpreted the data. V.I.F. provided theory support. K.W. and T.T. supplied hBN crystals A.M. and C.R.W. helped with experiments. M.B.S., J.R.P., V.I.F. and A.K.G. wrote the manuscript with input from all the authors.

\section{Additional information}

Supplementary information is available in the online version of the paper. Reprints and permissions information is available online at www.nature.com/reprints.

Correspondence and requests for materials should be addressed to V.I.F. or A.K.G.

\section{Competing financial interests}

The authors declare no competing financial interests. 


\section{Methods}

The measurements were carried out in a helium- 3 cryostat for $T$ down to $0.3 \mathrm{~K}$ and in a dilution refrigerator for lower $T$. All electrical connections to the sample passed through cold RC filters (Aivon Therma) and additional a.c. filters were on the top of the cryostats. The differential resistance was measured in the quasi-four-terminal geometry (using 4 superconducting leads to an SGS junction) and in the current-driven configuration using an Aivon preamplifier and a lock-in amplifier

To probe the superconducting proximity, we used an excitation current of $2 \mathrm{nA}$. 